\title{
DETERMINATION OF PHYSICAL PROPERTIES OF LAMINATED COMPOSITE BEAM VIA THE INVERSE VIBRATION PROBLEM METHOD
}

\author{
Murat Balcı ${ }^{1, *}$ and Ömer Gündoğdu ${ }^{2}$ \\ ${ }^{1}$ Bayburt University. Engineering Faculty. Mechanical Engineering, \\ Bayburt, Turkey. \\ Phone: +90-458-2111178 \\ *Email: mbalci@bayburt.edu.tr \\ ${ }^{2}$ AtatürkUniversity. Engineering Faculty. Mechanical Engineering, \\ Erzurum, Turkey. \\ Phone: +90-442-231-1111
}

\begin{abstract}
In this study, some physical properties of a laminated composite beam were estimated by using the inverse vibration problem method. Laminated composite plate was modeled and simulated to obtain vibration responses for different length-to-thickness ratios in ANSYS. A numerical model of the laminated composite beam with unknown parameters was also developed using a two-dimensional finite element model by utilizing the Euler-Bernoulli beam theory. Then, these two models were embedded into the optimization program to form the objective function to be minimized using genetic algorithms. After minimizing the squared difference of the natural frequencies from these two models, the unknown parameters of the laminated composite beam were found. It is observed in this study that the Euler-Bernoulli beam theory suppositions approximated the real results with a rate of $\% 0.026$ error as the thickness of the beam got thinner. The estimated values were finally compared with the expected values and a very good correspondence was observed.
\end{abstract}

Keywords: Inverse problem; finite element method; laminated composite beam; genetic algorithm; free vibration.

\section{INTRODUCTION}

The use of composites as engineering materials has increased greatly in recent years. Because of their strength, lightweight, resistance to corrosion and wear, and some other superior properties, they have taken the place of other engineering materials (Adebisi, Maleque, \& Rahman, 2011; Hariprasad, Dharmalingam, \& Praveen Raj, 2013). For this reason, the physical properties of composite materials need to be known in order to be analyzed and designed structurally. Composite beams find an important area of application in many mechanical, civil and aeronautical engineering structures (Giunta, Biscani, Belouettar, Ferreira, \& Carrera, 2013; Jeffrey, arlochan, \& Rahman, 2011; Umar, Zainudin, \& Sapuan, 2012). As a result, studies on their static and dynamic stability analysis have gained an important place among mechanics research, and hence a vast amount of study has been carried out on this area lately (Huzni et al., 2013; Li, $\mathrm{Wu}$, Kong, Li, \& Wu, 2014). However, designing composite beams to represent prespecified behavior or that are suitable for any working conditions is a hard task because of the large number of unknown parameters involved in their design. Consequently, to 
overcome this difficulty and to estimate composite beam parameters, the inverse vibration problem method has found its place in the design of composite beams.

The inverse vibration problem method can be basically used to estimate unknown parameters by using data obtained from experiments or computer simulations (Aster, Borchers, \& Thurber, 2013). A number of studies have used inverse vibration for parameter estimations. For instance, (Huang, Shih, \& Kim, 2009) attempted the inverse vibration method to solve a forced vibration problem that arose in cutting tools which were modeled as Euler-Bernoulli beams. In the numerical solutions, a conjugate gradient method was utilized and the simulation results for the beam displacements were used to estimate the external forces on the cutting tool. (Huang, 2005) tackled an inverse nonlinear forced vibration problem and solved it by using the conjugate gradient method. In the solution, experimental results were used to estimate external forces on a damped multi degree of freedom system. (Chiwiacowsky, de Campos Velho, \& Gasbarri, 2004) used the dynamics inverse problem to assess damage in buildings through the use of experimental vibration measurements. (Marinov \& Vatsala, 2008) utilized the variational imbedding method to solve the inverse problem that arose during the estimation of unknown coefficients of the Euler-Bernoulli equation. (Gladwell, 1997, 1999, 2006) developed a finite element model for an inline two-degrees-offreedom system and solved it as an inverse vibration problem. Mass and stiffness matrices were written in a closed form procedure in such a way as to minimize the mass.

The dynamic stability of composite Euler-Bernoulli beams was also investigated in a number of studies. For instance, (Della \& Shu, 2005) solved analytically the free vibration problem of composite beams with two overlapping delaminations. The problem was investigated with various boundary conditions and obtained natural frequency and mode shapes. Results from this model were compared with the experimental findings. (Wang, 2013) obtained a set of coupled linear differential equations of motion for free vibration of a composite beam with an asymmetric crosssection using the Euler-Bernoulli beam approach. An algorithm was developed to solve the resultant equations. (Ghayesh, Yourdkhani, Balar, \& Reid, 2010) investigated the vibration and stability of laminated composite beams. The governing equations were obtained using Newton's second law of motion and constitutive relations based on classical laminated beam theory. In this paper, the inverse vibration problem was utilized to find the physical properties of a laminated composite Euler-Bernoulli beam from its measured vibration frequencies. In the method proposed, the difference between the measured frequencies and the ones from a numerical model with unknown parameters is minimized to be able to choose the best solution among infinitely many possible solutions that can arise in an inverse method. Simulation results from an ANSYS model were used to imitate the experimental data. Genetic algorithms are used in the optimizations to avoid being trapped by local minima.

\section{MATHEMATICAL MODEL}

Basic assumptions of the Euler-Bernoulli beam theory are similar to the classical lamination theory. These theories are only valid for thin laminates. According to EulerBernoulli beam theory, the deformations caused by the transverse shear stresses are accepted as zero (Wang, Reddy, \& Lee, 2000). The bending behavior of an EulerBernoulli beam is shown in Figure 1.The axes and cross-section of the beam under consideration are shown in Figure 2. The letter $L$ represents the beam length, $I$ the area 
moment of inertia, $A$ the cross sectional area, $b$ the width of the beam, and $h$ the height of the beam.

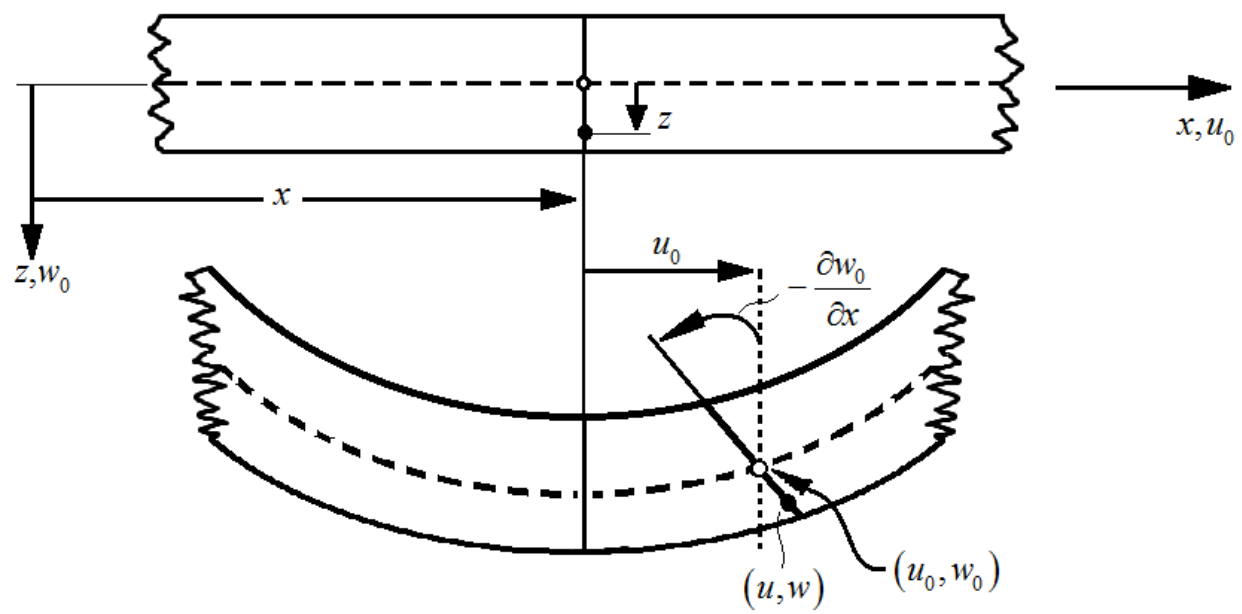

Figure 1. Euler-Bernoulli beam theory (Wang et al., 2000).

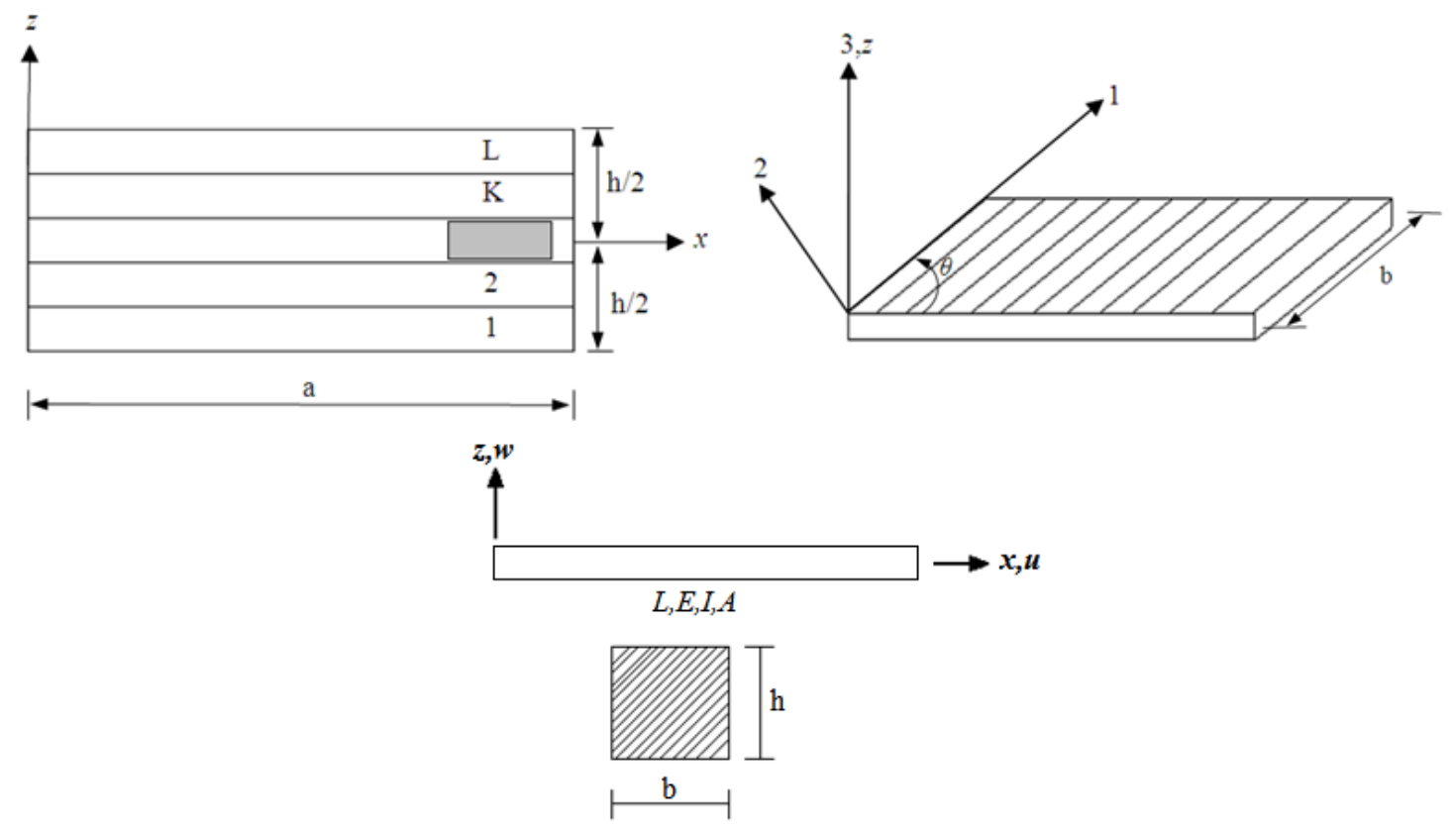

Figure 2. Coordinates and geometry of laminated composite beam.

The stress-strain relationship for a laminated composite Euler-Bernoulli beam is given by Hooke's law as in Eq. (.1).

$$
\{\sigma\}=[D]\{\varepsilon\}
$$

where $[D]$ is the bending stiffness matrix while $\sigma$ represents the stress. The material was assumed to be linear orthotropic.

The terms in Eq. (Chandra, Singh, \& Gupta) are defined as follows (Kollár \& Springer, 2003): 


$$
\begin{gathered}
\{\sigma\}^{i}=\left[\begin{array}{lll}
\sigma_{x} & \sigma_{y} & \tau_{x y}
\end{array}\right]^{T^{i}} \\
\{\varepsilon\}^{i}=\left\{\begin{array}{lll}
\varepsilon_{x} & \varepsilon_{y} & \gamma_{x y}
\end{array}\right\}^{T^{i}} \\
{[Q]^{i}=\left[\begin{array}{ccc}
Q_{11} & Q_{12} & 0 \\
Q_{12} & Q_{22} & 0 \\
0 & 0 & Q_{66}
\end{array}\right]^{i}}
\end{gathered}
$$

where $[Q]^{i}$ represents the reduced elastic constants matrix for the $\mathrm{i}^{\text {th }}$ layer, as given in Eq. (4) (Reddy, 2003):

$$
\begin{aligned}
& Q_{11}=\frac{E_{1}}{1-v_{12} v_{21}}, \quad Q_{12}=\frac{v_{12} E_{2}}{1-v_{12} v_{21}} \\
& Q_{22}=\frac{E_{2}}{1-v_{12} v_{21}}, \quad Q_{66}=G_{12}
\end{aligned}
$$

Stiffness matrices for a composite plate composed of $L$ layers (Balci, 2011) are given as

$$
\begin{aligned}
& {[A]=\int_{-h_{b}}^{h_{t}}[Q] d z} \\
& {[B]=\int_{-h_{b}}^{h_{t}} z[Q] d z} \\
& {[D]=\int_{-h_{b}}^{h_{t}} z^{2}[Q] d z}
\end{aligned}
$$

The $[A],[B],[D]$ matrices are the extensional stiffness, bending-extensional coupling stiffness, and bending stiffness matrices, respectively. The bendingextensional coupling stiffness matrix for composite plates with symmetric laminate is given as $[B]=0$ (Balci, 2011). Due to symmetry, the strains with respect to the reference axis are $\varepsilon_{x}^{0}, \varepsilon_{y}^{0}, \gamma_{x y}^{0}=0$ (Balci, 2011).

\section{FINITE ELEMENT MODEL}

A planar beam bending element with two nodes, each having two degrees of freedom, was chosen in accordance with the Euler-Bernoulli beam theory. The beam element, having the same degree of freedom as the beam deflection $w$ and the rotation of the cross-section $\theta$,is depicted in Figure 3.

The potential energy for a beam element in bending vibration is given as (Petyt, 1990)

$$
U=\frac{1}{2} \int_{0}^{l} E I\left(\frac{d^{2} w}{d x^{2}}\right)^{2} d x
$$




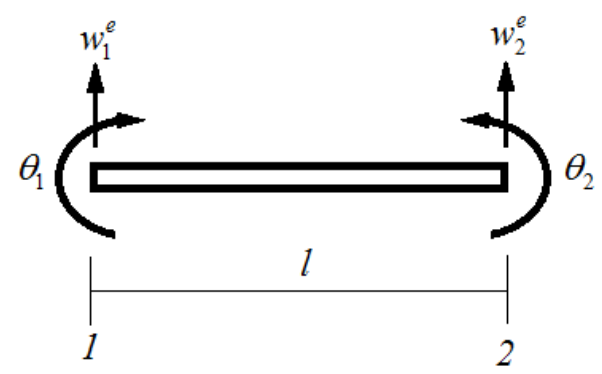

Figure 3. Plane beam bending element.

The kinetic energy of a beam element in bending vibration is

$$
T=\frac{1}{2} \int_{0}^{l} \rho A\left(\frac{d w}{d t}\right)^{2} d x
$$

If the potential and kinetic energy expressions are substituted in the Hamilton principle (Petyt, 1990),

$$
L=\int_{t_{0}}^{t_{1}} \delta(T-U) d t+\int_{t_{0}}^{t_{1}} \delta W d t
$$

is obtained, where $W$ expresses the work done by external forces. If Eq. (9) is minimized for a system under undamped free vibration, the equation of motion of a laminated composite beam undergoing bending vibrations can be obtained as (Balci, 2011)

$$
\left[m^{e}\right]\left\{\ddot{q}^{e}\right\}+\left[k^{e}\right]\{q\}=0
$$

where $\left[m^{e}\right]$ and $\left[k^{e}\right]$ are the mass and stiffness matrices, respectively. If the curvature of the laminated composite beam is re-written,

$$
\kappa=\frac{d^{2} w}{d x^{2}}=\frac{d^{2}}{d x^{2}}\{w\} \rightarrow \kappa=\boldsymbol{D} w
$$

Where $\boldsymbol{D}$ is the linear differential operator and $\boldsymbol{w}$ displacement. The beam displacement equation is then (Kollár \& Springer, 2003)

$$
w=N d
$$

Where $\boldsymbol{N}$ represents the shape function and $\boldsymbol{d}$ the nodal displacement operator. The nodal displacement operator is given as

$$
\boldsymbol{d}=\left\{\begin{array}{c}
w_{i} \\
\frac{d w_{i}}{d x}
\end{array}\right\}=\left\{\begin{array}{c}
w_{i} \\
\theta_{i}
\end{array}\right\}
$$


If Eq. (13) is substituted into Eq. (12), the expression

$$
\kappa=D w=D N d \rightarrow \kappa=B d
$$

isobtained (Kollár \& Springer, 2003), where the expression $\boldsymbol{B}$ is the strain-displacement matrix. If the stiffness matrix for the bending beam element is given as (Petyt, 1990),

$$
\left[k^{e}\right]=E I \int_{A} \boldsymbol{B}^{T} \boldsymbol{B} d A
$$

the mass matrix for the bending beam element is also given as (Petyt, 1990),

$$
\left[m^{e}\right]=\rho A \int_{A} N^{\mathrm{T}} N d x
$$

If the mass and stiffness matrices developed for the bending beam element are combined together so as to represent a laminated composite beam,

$$
\begin{aligned}
& {[M]=\sum_{e=1}^{n} m^{e}} \\
& {[K]=\sum_{e=1}^{n} k^{e}}
\end{aligned}
$$

are obtained, where the matrices $[\mathrm{M}]$ and $[\mathrm{K}]$ are respectively the global mass and stiffness matrices, and $n$ is the number of finite elements used in the model.

\section{DEVELOPMENT OF SHAPE FUNCTION}

The plain beam element shown in Figure 3 has two nodes with two degrees of freedom, and hence it has four degrees of freedom in total. To form the shape function, a cubic polynomial with four terms for each degree of freedom has been chosen as the displacement shape function:

$$
w(x)=a_{1}+a_{2} x+a_{3} x^{2}+a_{4} x^{3}
$$

The beam displacement can be written in the following form (De Abreu, Ribeiro, \& Steffen Jr, 2004):

$$
w=\{P\}^{\mathrm{T}}\{a\}
$$

In Eq. (19), $a$ is the coefficient vector and $P$ is the interpolation polynomial term vector. The coefficient and the interpolation polynomial term vector are given in the following format (De Abreu et al., 2004):

$$
\begin{aligned}
& \{a\}=\left\{\begin{array}{llll}
a_{1} & a_{2} & a_{3} & a_{4}
\end{array}\right\}^{\mathrm{T}} \\
& \{P\}=\left\{\begin{array}{llll}
1 & x & x^{2} & x^{3}
\end{array}\right\}^{\mathrm{T}}
\end{aligned}
$$


If the displacement polynomial given in Eq. (18) is substituted into Eq. (12) in a matrix form and expanded for each node (De Abreu et al., 2004),

$$
\{\boldsymbol{d}\}=[X]\{a\}
$$

is obtained. $X$ in Eq. (21) represents the expanded displacement matrix of dimension $4 \times 4$. Then, Eq. (21) is solved for the coefficient vector:

$$
\{a\}=[X]^{-1}\{\boldsymbol{d}\}
$$

If Eq. (22) is substituted intoEq. (19), one may obtain

$$
w=\{P\}^{\mathrm{T}}[X]^{-1}\{\boldsymbol{d}\}
$$

If Eq. (23) is substituted intoEq. (12) and reorganized, the shape function is developed in the form below:

$$
\boldsymbol{N}=\{P\}^{\mathrm{T}}[X]^{-1}
$$

\section{DYNAMICS ANALYSIS}

The equation of motion for the beam undergoing an undamped free vibration was given in Eq. (10). The equation of motion for the global system is

$$
[M]\{\ddot{q}\}+[K]\{q\}=0
$$

for which a harmonic solution can be proposed in the following form:

$$
\{q\}=\{\psi\} \sin (\omega t)
$$

If Eq. (26) is substituted intoEq. (25)

$$
-[M]\{\psi\} \omega^{2} \sin (\omega t)+[K]\{\psi\} \sin (\omega t)=0
$$

can be obtained. If Eq. (27) is further reorganized, it takes the following eigenvalue problem form:

$$
\left([K]-\omega^{2}[M]\right)\{\psi\}=0
$$

where $\lambda=\omega^{2}$ are eigenvalues representing vibration frequencies while $\psi$ are eigenvectors representing vibration modes.

\section{FORMING OBJECTIVE FUNCTION FOR GENETIC ALGORITHM USE}

There are infinitely many possible solutions in the solution of inverse problems, and hence some form of optimization is necessary to choose the best solution amongst them. Furthermore, there is a possibility of getting trapped in local minima in such 
optimizations of multimodal problems. Therefore, genetic algorithms are utilized to make sure that global minima are searched for the solutions. Objective functions are needed in the optimizations, and the sum of squared difference between the frequencies obtained from simulations and numerical model was accepted as the objective function for these optimizations. Natural frequencies are chosen as optimization parameters, as they provide more information about systems with fewer data, and this also requires less computation time. The objective function used in the objective function evaluations is

$$
F F(t)=\min \sum_{i=1}^{n}\left(\left\{\omega_{\text {ANSYS }}(t)\right\}-\left\{\omega_{\text {Model }}(t)\right\}\right)^{2}
$$

where $\omega_{\text {ANSYS }}$ represents the natural frequencies obtained from ANSYS, which imitates experimental data, while $\omega_{\text {Model }}$ represents the frequencies obtained from the numerical model, which includes unknown system parameters.

In the optimizations with genetic algorithms, settings are of great importance because small changes result in large differences in the solutions. The settings were decided after a long period of trial and error, as shown in Table 1 (Balci, 2011).

Table 1.Genetic Algorithm Settings

\begin{tabular}{ll}
\hline Population size & 30 \\
Selection & Stochastic uniform \\
Mutation & Adaptive feasible \\
Mutation rate & 0.01 \\
Crossover & Heuristic \\
\hline
\end{tabular}

\section{RESULTS AND DISCUSSION}

\section{Simulation Data}

In the study performed in ANSYS, a laminated composite beam with four layers having [0/90/90/0] orientation angles and 1500x1500 mesh size is modeled with a clamped-free (Chirn \& McFarlane) boundary condition. In the model, a SHELL99 element was used for the laminated composite beam. The SHELL99 element uses governing equations based on the Timoshenko beam theory, which takes into account rotational inertia and shear deformation. With these capabilities, the SHELL99 element gives reasonable results in the analysis of linear thin structures. The geometric and physical properties of the laminated composite beam to be used in ANSYS are provided in Table 2.

Table 2.Geometric and physical properties of laminated composite beam (De Abreu et al., 2004).

\begin{tabular}{lll}
\hline$E_{x}=38 \mathrm{GPa}$ & $E_{y}=3.8 \mathrm{GPa}$ & $E_{z}=3.8 \mathrm{GPa}$ \\
$G_{x y}=1.9 \mathrm{GPa}$ & $G_{x z}=1.9 \mathrm{GPa}$ & $G_{y z}=0.76 \mathrm{GPa}$ \\
$v_{x y}=0.25$ & $v_{y z}=0.25$ & $\rho=1295 \mathrm{~kg} / \mathrm{m}^{3}$ \\
$L_{x}=1 \mathrm{~m}$ & $b=0.01 \mathrm{~m}$ & \\
\hline
\end{tabular}


The laminated composite plate was assumed to have a different length-tothickness ratio $\left(L_{x} / h\right)$ every time that the simulations were executed in ANSYS.

Table 3.Natural frequencies of laminated composite beamfrom simulations $(\mathrm{Hz})$

\begin{tabular}{cccccccc}
\hline \multirow{2}{*}{$L_{x} / h$} & \multicolumn{6}{c}{ Free vibration natural frequencies $(\mathrm{Hz}),\left(\omega_{\text {ANSYS }}\right)$} \\
\cline { 2 - 7 } & Mode I & Mode II & Mode III & Mode IV & Mode V & Mode VI & Mode VII \\
\hline 100 & 8.2646 & 51.611 & 143.70 & 279.31 & 456.95 & 674.13 & 928.05 \\
200 & 4.1342 & 25.885 & 72.377 & 141.53 & 233.34 & 347.42 & 483.37 \\
300 & 2.7563 & 17.267 & 48.317 & 94.594 & 156.18 & 232.97 & 324.81 \\
400 & 2.0673 & 12.953 & 36.255 & 71.009 & 117.30 & 175.08 & 244.30 \\
500 & 1.6539 & 10.363 & 29.011 & 56.830 & 93.903 & 140.20 & 195.69 \\
\hline
\end{tabular}

\section{Parameter Estimation}

The numerical model for the laminated composite beam was constructed in MATLAB, and the optimizations were realized in the Genetic Algorithm Toolbox in MATLAB. The finite element model for the laminated composite beam was constructed with a mesh size of $1500 \times 1500$, and its elasticity module $E$ and density $\rho$ were estimated for different boundary conditions. The estimates are tabulated together with the objective function evaluations and percent errors.

Table 4.Natural frequency $(\mathrm{Hz})$ estimates for laminated composite beam

\begin{tabular}{cccccccc}
\hline \multirow{2}{*}{$L_{x} / h$} & \multicolumn{7}{c}{ Natural frequencies (Hz) } \\
\cline { 2 - 8 } & Mode I & Mode II & Mode III & Mode IV & Mode V & Mode VI & Mode VII \\
\hline 100 & 7.9194 & 49.6171 & 138.9293 & 272.2459 & 450.0420 & 672.2849 & 938.9764 \\
200 & 4.0862 & 25.6079 & 71.7028 & 140.5088 & 232.2711 & 346.9728 & 484.6148 \\
300 & 2.7399 & 17.1704 & 48.0777 & 94.2131 & 155.7409 & 232.6500 & 324.9409 \\
400 & 2.0635 & 12.9317 & 36.2091 & 70.9554 & 117.2943 & 175.2174 & 244.7252 \\
500 & 1.6509 & 10.3455 & 28.9678 & 56.7654 & 93.8372 & 140.1766 & 195.7838 \\
\hline
\end{tabular}

Table 5.Natural frequency estimates for laminated composite beam for different " $L_{x} / h$ "

\begin{tabular}{cccccccc}
\hline$L_{x} / h$ & \multicolumn{2}{c}{$\begin{array}{c}\text { Desired } \\
\left(\times 10^{9} \mathrm{~Pa}\right)\end{array}$} & \multicolumn{2}{c}{$\begin{array}{c}\text { Estimated } \\
\left(\times 10^{9} \mathrm{~Pa}\right)\end{array}$} & \multicolumn{2}{c}{$\%$ Error } & \multirow{2}{*}{$\begin{array}{c}\text { Objective } \\
\text { function } \\
\text { evaluations }\end{array}$} \\
\cline { 2 - 6 } & $E_{x}$ & $E_{y}$ & $E_{x}$ & $E_{y}$ & $E_{x}$ & $E_{y}$ & 247.2675 \\
100 & 38 & 3.8 & 34.842 & 4.192 & 8.484 & -10.263 & 4.4689 \\
200 & 38 & 3.8 & 37.189 & 4.0291 & 2.134 & -6.029 & 0.5243 \\
300 & 38 & 3.8 & 37.644 & 3.9161 & 0.937 & -3.0552 & 0.2051 \\
400 & 38 & 3.8 & 37.989 & 3.755 & 0.029 & 1.184 & 0.020 \\
500 & 38 & 3.8 & 37.990 & 3.76 & 0.026 & 1.053 & 0 \\
\hline
\end{tabular}




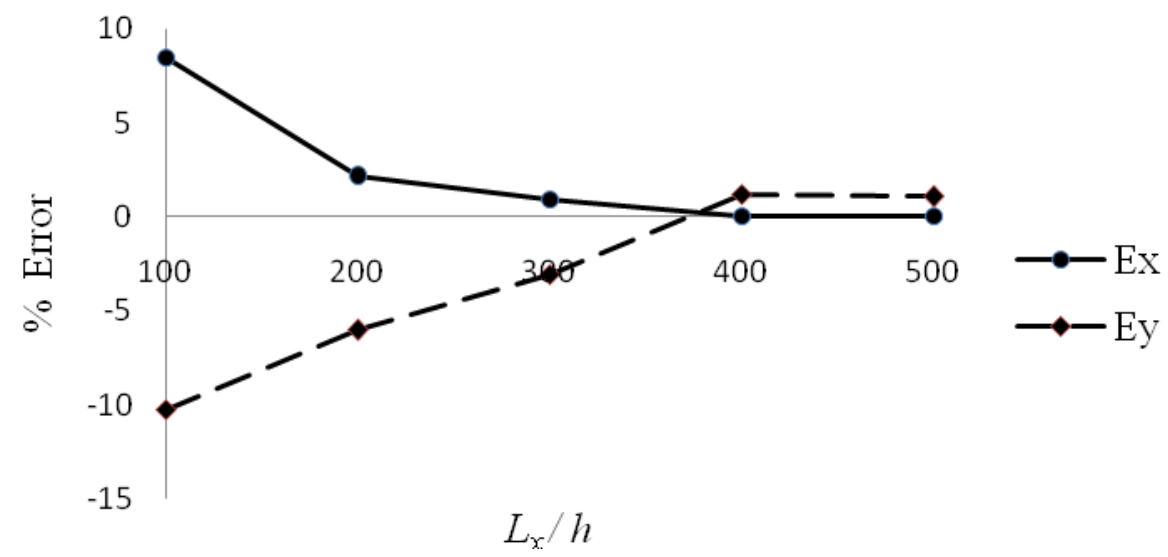

Figure 4. Variation of \% error for $E_{x}$ and $E_{y}$ with respect to " $L_{x} / h$ ".

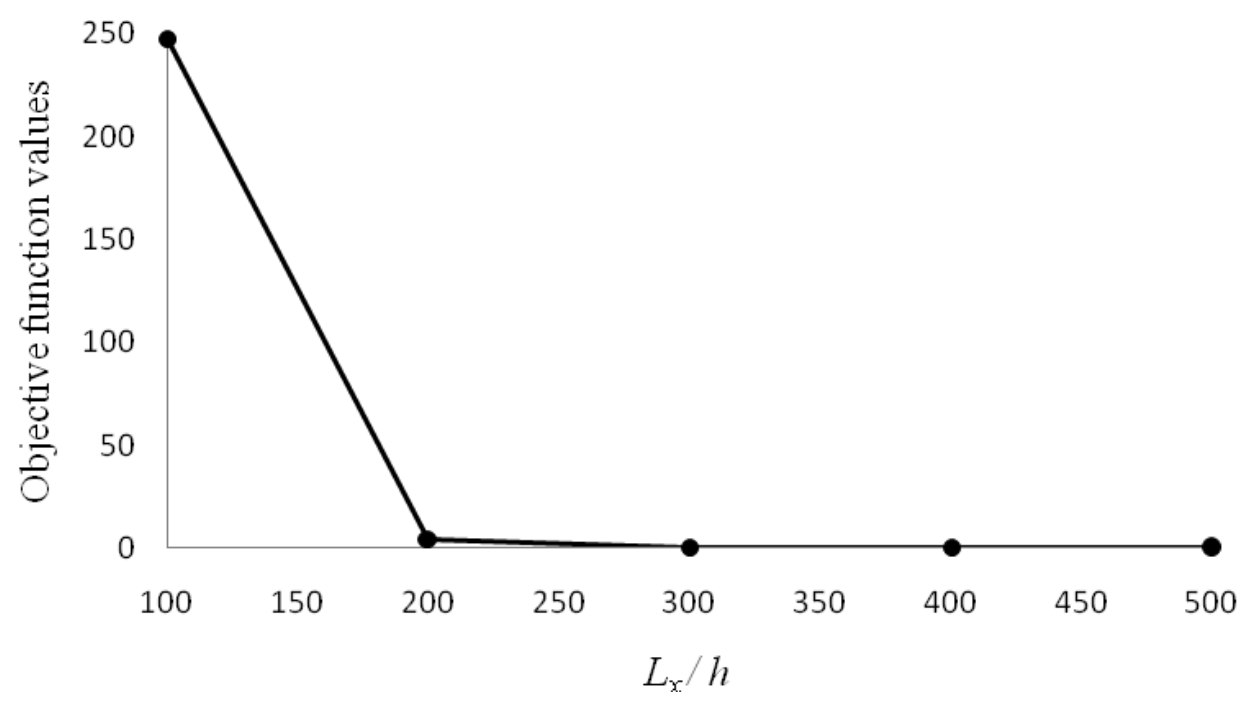

Figure 5. Variation of objective function evaluations for $E_{x}$ and $E_{y}$ with respect to

$$
\text { " } L_{x} / h " \text {. }
$$

In this study, the physical properties of an Euler-Bernoulli beam were estimated based on its known/measured natural frequencies. The simulation results obtained from ANSYS were assumed to be experimental data. On the other hand, the numerical model with unknown parameters was established in MATLAB. Finally, the natural frequencies from these two sources were combined in the objective function to estimate the unknown physical parameters. The inverse vibration problem was tackled as an optimization problem and solved using the MATLAB Genetic Algorithm Toolbox. Elasticity modules $\left(E_{x}\right.$ and $\left.E_{y}\right)$ of the beam were estimated simultaneously for different rates of length-thickness conditions and compared with the real values. In the computations for the two-parameter estimation problem in the $x$ and $y$ directions, the objective function evaluations approached zero while the percent errors in the $x$ and $y$ directions were, respectively, observed to stay constant around $0.026 \%$ and $1.053 \%$ as the length-to-thickness ratio increased. 


\section{CONCLUSIONS}

The proposed method gives a basis not only for estimation of physical properties of materials used in a system but also for estimation of initial conditions and/or boundary conditions, etc. When the results obtained from the optimizations are compared with the real values, very good correspondence is observed within a maximum error of $10 \%$. Although the result would be satisfactory for most applications, further improvements might be sought to improve the following items:

i) A lamination theory of first or higher degrees, which takes the shear deformations into account, could be used.

ii) The damping effect could be added to the model.

iii) A nonlinear analysis could be performed.

iv) Different finite-elements with appropriate shape functions could be used to improve the FEM analysis.

v) The objective function in the genetic algorithm part could be modified to include some other parameters other than just natural frequencies, or constraints, to improve the optimization results.

vi) The genetic algorithm parameters themselves, such as mutation, crossover, etc.,could be adjusted or supported with some intelligence like fuzzy and/or neural nets to get more effective solutions in the optimization.

\section{REFERENCES}

Adebisi, A. A., Maleque, M. A., \& Rahman, M. M. (2011). Metal matrix composite brake rotor: Historical development and product life cycle analysis. International Journal of Automotive and Mechanical Engineering, 4, 471-480.

Aster, R. C., Borchers, B., \& Thurber, C. H. (2013). Parameter estimation and inverse problems. USA: Academic Press.

Balci, M. (2011). Estimation of physical properties of laminated composites via the method of inverse vibration problem. (Ph.D), Ataturk University, Erzurum.

Chandra, R., Singh, S. P., \& Gupta, K. (1999). Damping studies in fiber-reinforced composites-a review. Composite structures, 46(1), 41-51.

Chirn, J. L., \& McFarlane, D. C. (2000, 2000). A holonic component-based approach to reconfigurable manufacturing control architecture. Paper presented at the Database and Expert Systems Applications, 2000. Proceedings. 11th International Workshop on.

Chiwiacowsky, L. D., de Campos Velho, H. F., \& Gasbarri, P. (2004). A variational approach for solving an inverse vibration problem. Paper presented at the Inverse Problems, Design and Optimization Symposium, Rio de Janeiro, Brazil.

De Abreu, G., Ribeiro, J., \& Steffen Jr, V. (2004). Finite element modeling of a plate with localized piezoelectric sensors and actuators. Journal of the Brazilian Society of Mechanical Sciences and Engineering, 26(2), 117-128.

Della, C. N., \& Shu, D. (2005). Free vibration analysis of composite beams with overlapping delaminations. European Journal of Mechanics-A/Solids, 24(3), 491-503.

Ghayesh, M. H., Yourdkhani, M., Balar, S., \& Reid, T. (2010). Vibrations and stability of axially traveling laminated beams. Applied Mathematics and Computation, 217(2), 545-556. 
Giunta, G., Biscani, F., Belouettar, S., Ferreira, A., \& Carrera, E. (2013). Free vibration analysis of composite beams via refined theories. Composites Part B: Engineering, 44(1), 540-552.

Gladwell. (1997). Inverse vibration problems for finite-element models. Inverse Problems, 13(2), 311.

Gladwell. (1999). Inverse finite element vibration problems. Journal of Sound and Vibration, 221(2), 309-324.

Gladwell. (2006). Minimal mass solutions to inverse eigenvalue problems. Inverse Problems, 22(2), 539.

Hariprasad, T., Dharmalingam, G., \& Praveen Raj, P. (2013). A study of mechanical properties of banana-coir hybrid composite using experimental and fem techniques. Journal of Mechanical Engineering and Sciences, 4, 518- 531.

Huang, C.-H. (2005). A nonlinear inverse problem in estimating simultaneously the external forces for a vibration system with displacement-dependent parameters. Journal of the Franklin Institute, 342(7), 793-813.

Huang, C.-H., Shih, C.-C., \& Kim, S. (2009). An inverse vibration problem in estimating the spatial and temporal-dependent external forces for cutting tools. Applied Mathematical Modelling, 33(6), 2683-2698.

Huzni, S., Ilfan, M., Sulaiman, T., Fonna, S., Ridha, M., \& Arifin, A. K. (2013). Finite element modeling of delamination process on composite laminate using cohesive element. International Journal of Automotive and Mechanical Engineering, 7, 1023-1030.

Jeffrey, K. J. T., arlochan, F., \& Rahman, M. M. (2011). Residual strength of chop strand mats glass fiber/epoxy composite structures: Effect of temperature and water absorption. International Journal of Automotive and Mechanical Engineering, 4, 504-519.

Kollár, L. P., \& Springer, G. S. (2003). Mechanics of composite structures: Cambridge university press.

Li, J., Wu, Z., Kong, X., Li, X., \& Wu, W. (2014). Comparison of various shear deformation theories for free vibration of laminated composite beams with general lay-ups. Composite structures, 108, 767-778.

Marinov, T. T., \& Vatsala, A. S. (2008). Inverse problem for coefficient identification in the euler-bernoulli equation. Computers \& Mathematics with Applications, 56(2), 400-410.

Petyt, M. (1990). Introduction to finite element vibration analysis: Cambridge university press.

Reddy, J. N. (2003). Mechanics of laminated composite plates and shells: Theory and analysis: CRC press.

Umar, A. H., Zainudin, E. S., \& Sapuan, S. M. (2012). Effect of accelerated weathering on tensile properties of kenaf reinforced high-density polyethylene composites. Journal of Mechanical Engineering and Sciences, 2, 198-205.

Wang, C. M., Reddy, J. N., \& Lee, K. H. (2000). Shear deformable beams and plates: Relationships with classical solutions. Oxford: Elsevier.

Wang, G. (2013). Coupled free vibration of composite beams with asymmetric crosssections. Composite structures, 100(0), 373-384. 